Classification

Physics Abstracts

$52.25-52.80-52.50$

\title{
Calculation of the population density in the low pressure mercury-argon discharge : analysis of the steady state and sinusoidal operating modes of the positive column
}

\author{
V. Plagnol, G. Zissis, I. Bernat, J. J. Damelincourt and J. L. Bonneval \\ Laboratoire des Décharges dans les Gaz (U.R.A. 277), Unversité Paul Sabatier, 118 rte de \\ Narbonne, 31062 Toulouse, France
}

(Received 26 May 1992, revised 13 January 1993, accepted 20 January 1993)

\begin{abstract}
This work deals with the setting up of a collisional-radiative model concerning low pressure mercury-argon discharge operating under different power supplies (dc and ac). This model allows determination of various characteristic quantities of the discharge using the population and electronic energy conservation equations. In view of optimisation of the consuming computer time, some simplifying but still realistic hypotheses have been assumed. A first study in the frame of the steady state (dc), allows us to evaluate the «weight » of certain physical phenomena in this type of modelling. We assess how much it is of prime importance to consider $\mathrm{Hg}-\mathrm{Hg}$ collisions as well as the excitation and ionization of argon in discharge plasmas. The first point, often discarded by many authors, has however a quite appreciable influence on the different discharge parameters. Besides, the determination of the escape factors for the $\mathrm{Hg}$ resonance lines at $254 \mathrm{~nm}$ and $185 \mathrm{~nm}$ are not for the least insignificant.
\end{abstract}

\section{Introduction.}

Lots of experimental and theoretical studies concerning low pressure mercury-argon discharge exist. The main application of such studies deals with lighting domain, in particular with the fluorescent tube that is a specific low pressure mercury-argon discharge. The last few years, important progresses, concerning not only the increase of light efficiency but more generally better knowledge about gas discharge behaviour, have been achieved. These researches mainly turn to more and more complete models which allow the prediction of optimal operating conditions of lamps. Thus, better understanding of important mechanisms taking place in the system is accomplished as well.

This work deals with the setting up of a collisional-radiative model concerning low pressure mercury-argon discharges operating under different excitation mode (dc and ac).

The present paper discusses results of numerical calculations on the $\mathrm{Hg}$ - Ar positive column. A first study in the frame of the steady state (at constant current), allows evaluation of the weight of certain physical phenomena in this type of discharge. We have considered inelastic neutral-neutral collisions as well as the excitation and ionization of argon in discharge plasma. 
The determination of the escape factors for the $\mathrm{Hg}$ resonance lines at $254 \mathrm{~nm}$ and $185 \mathrm{~nm}$ have been approached by several ways (Holstein's theory, Bernat's calculations and experiment data).

It is known from experiments that the efficiency of the production of the $\mathrm{Hg}$ resonance radiation goes up with increasing supply frequency [1]. Many authors have tried to explain this phenomenon that is still not so clearly understood. In the case of ac excitation, we deal with a more complete study on the frequency dependence of the main characteristics of the plasma, namely excited state density populations, electric field, electron density and temperature..

All the results are obtained under the reference conditions illustrated in table $\mathrm{I}$.

Table I. - Input data for calculations in the dc and ac cases.

\begin{tabular}{c|c|c}
\hline \hline Parameters & $\begin{array}{c}\text { Steady-State } \\
\text { Operation }\end{array}$ & $\begin{array}{c}\text { Sinusoidal } \\
\text { Operation }\end{array}$ \\
\hline Tube radius (mm) & & 18 \\
Ar pressure (Torr) & \multicolumn{2}{|c|}{3} \\
Filling Temperature $\left({ }^{\circ} \mathrm{C}\right)$ & $10 \rightarrow 60$ & 24 \\
Tube Cold spot Temperature $\left({ }^{\circ} \mathrm{C}\right)$ & - & 40 \\
Operating Frequency (Hz) & 400 & $50 \rightarrow 20 \times 10^{3}$ \\
Electric Current (mA) & $400 \sqrt{2} \sin (2 \pi v \mathrm{t})$ \\
\hline \hline
\end{tabular}

In this paper the following notations are used for the external discharge parameters : $I$ is the current; $\omega=2 \pi \nu$ is the pulsation of the signal and $\nu$ the frequency; $R$ is the radius of the discharge tube; $P_{\mathrm{Ar}}$ is the argon pressure; $T_{\mathrm{cs}}$ is the cold spot temperature. We note that the cold spot temperature fixes the mercury saturated vapour pressure in the discharge.

\section{Theoretical model.}

The theoretical analysis of $\mathrm{Hg}$-rare gas discharges being very complex, some simplifying but still realistic hypotheses have been assumed in order to optimize the consuming computer time.

The positive column is assumed to be axisymmetric and therefore all calculations have been performed using cylindrical co-ordinates.

In this model, we consider a six level scheme for mercury as illustrated in figure 1 , where $n_{x}, x \in\left\{\mathrm{g}, \mathrm{m}, \mathrm{r}, \mathrm{s}, \mathrm{p}, \mathrm{u}, \mathrm{Hg}^{+}\right\}$, denotes respectively the population density of the following mercury atomic states : $6^{1} \mathrm{~S}_{0}$ (ground), $6{ }^{3} \mathrm{P}_{0,1,2}, 6^{1} \mathrm{P}_{1}, 7^{3} \mathrm{~S}_{1}$ (excited levels) and the mercury ion. The excited state densities of the $\mathrm{Hg}\left(6^{3} \mathrm{P}\right)$ atomic levels are very important for this type of studies because the luminous emitted power at $254 \mathrm{~nm}$ depends directly on them.

In the low pressure discharges, a diffusion controlled positive column is frequently assumed (Schottky's regime). The main ion-electron loss process is the diffusion of these pairs on the tube wall where recombination takes place, rather than the volume recombination. The formation of molecular ion $\mathrm{Hg}_{2}^{+}$can be neglected. This hypothesis is confirmed by the fact that this molecular ion is very unstable (the corresponding dissociating energy being $0.06 \mathrm{eV}$ ).

Several authors assume that, the argon's role is limited to that of a buffer gas. This means that only the mercury can be excited and ionized. However, it seems that argon not only controls the electron mobility in the discharge but it is also an active species, therefore Ar 


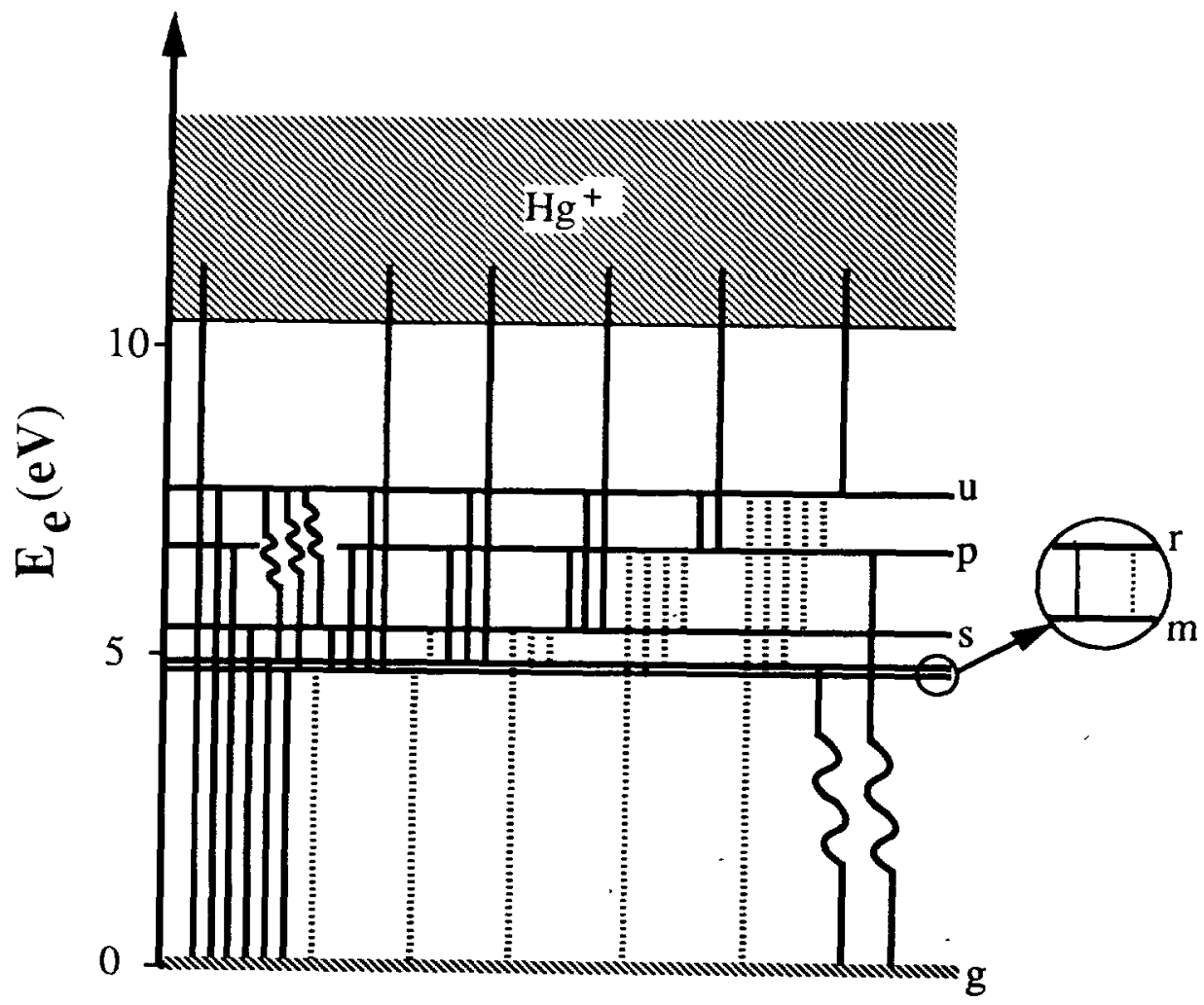

Fig. 1. - Simplified mercury atomic structure used in this work. Solid lines represent electron-atom inelastic collisions, dashed lines correspond to the 2 nd kind collisions and the net radiative transitions are represented by wavy lines.

excitation and ionization are not negligible. In this paper we consider a very simplified argon atomic structure which includes an excitation and an ionization state as shown in figure 2 ; Ar* is a fictive level which regroups the first two radiative levels and the first two metastable levels $\left(1 \mathrm{~s}_{2}, 1 \mathrm{~s}_{3}, 1 \mathrm{~s}_{4}\right.$ and $\left.1 \mathrm{~s}_{5}\right)$. The average energy of these atomic levels is about $11.65 \mathrm{eV}$. However, direct ionization from the ground state is assumed negligible under the conditions considered in this study (the mean electron energy is about $1-2 \mathrm{eV}$ ).

Interactions such as quenching, associative ionization.. between argon atoms are neglected because of its low excited state concentration. We also neglect inelastic interactions between excited argon and mercury atoms because of the very small value of atomic ratio $\left.\left[\mathrm{Ar}^{*}\right] / \mathrm{Hg}\right]$ (about $10^{-4}-10^{-5}$ ). However, we consider the following inelastic reaction :

$$
\mathrm{Hg}\left(6^{3} \mathrm{P}_{2}\right)+\mathrm{Hg}\left(6^{3} \mathrm{P}_{2}\right) \rightarrow \mathrm{Hg}_{2}^{+}+\mathrm{e}^{-}
$$

This reaction corresponds to an associative ionization process and should be considered because of its destructive contribution to the population of the $\mathrm{Hg}\left(6{ }^{3} \mathrm{P}_{2}\right)$ level, thus influencing directly the $\mathrm{Hg}$ resonance line production.

Since excitation and ionization characteristic times are lower than the diffusion ones, we neglect these terms in the relevant continuity equations.

The electronic temperature is assumed constant over the discharge tube radius. This assumption is justified experimentally by probe measurements. Furthermore, more complete 


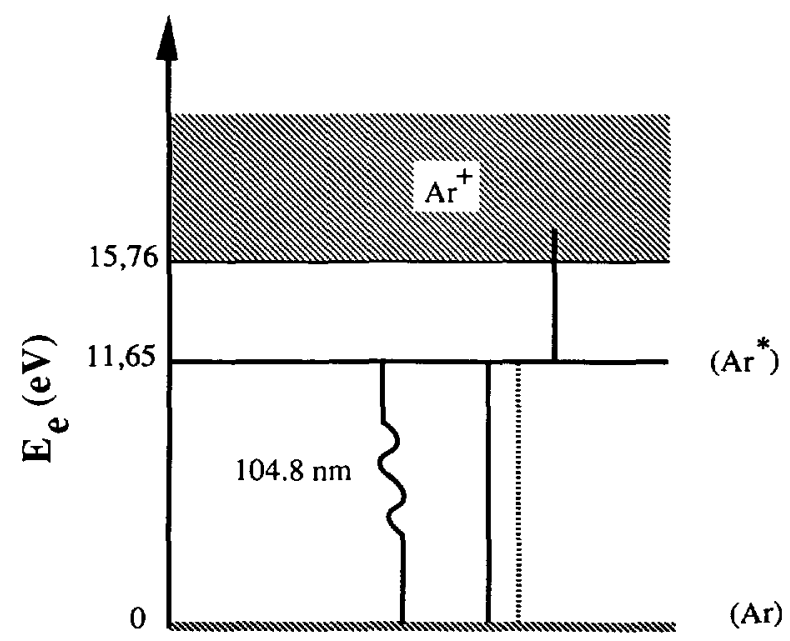

Fig. 2. - Simplified argon atomic structure used in this work. We used the same notation as in figure 1.

calculations have shown that the radial variation of the heavy gas temperature remains, in our cases, less than $20 \%$, therefore we consider a flat radial heavy gas temperature distribution. Under this last assumption, $\mathrm{Hg}$ and $\mathrm{Ar}$ ground states are supposed to be uniformly distributed within the discharge tube. We then, deduce $n_{\mathrm{Hg}}$ and $n_{\mathrm{Ar}}$ from the perfect gas law:

$$
\begin{aligned}
& n_{\mathrm{Hg}}=\frac{P_{\mathrm{Hg}}\left(T_{\mathrm{cs}}\right)}{k_{\mathrm{B}} T_{\mathrm{g}}} \\
& n_{\mathrm{Ar}}=\frac{P_{\mathrm{Ar}}\left(T_{\mathrm{g}}\right)}{k_{\mathrm{B}} T_{\mathrm{g}}}
\end{aligned}
$$

$P_{\mathrm{Hg}}\left(T_{\mathrm{cs}}\right)$ is the mercury saturated vapour pressure and it is only a function of the cold spot temperature ; $k_{\mathrm{B}}$ is the Boltzmann constant and $T_{\mathrm{g}}$ the temperature of neutral atoms, obtained by averaging the radial values given by Zissis [3]. Taking into account that the discharge tube is a closed system, $P_{\mathrm{Ar}}\left(T_{\mathrm{g}}\right)$ can be deduced from the following equation :

$$
P_{\mathrm{Ar}}\left(T_{\mathrm{g}}\right)=P_{\mathrm{Ar}}^{0} \frac{T_{\mathrm{g}}}{T_{0}}
$$

$T_{0}$ is the tube filling temperature and $P_{\mathrm{Ar}}^{0}$ is the argon pressure at this temperature.

The radial behaviour of the charged and excited species is more complicated and should be evaluated by solving the governing equation set at each radial node. However, several authors use a radial distribution of the type :

$$
n_{x}(r)=n_{x}(0) J_{0}\left(2.4 \frac{r}{R}\right) \quad x \in\left\{\mathrm{m}, \mathrm{r}, \mathrm{s}, \mathrm{p}, \mathrm{u}, \mathrm{Hg}^{+}, \mathrm{e}^{-}\right\}
$$

where $J_{0}$ is the zero-order Bessel function. Calculations carried out by several authors, using this distribution are in very good agreement (within $\pm 2 \%$ ) with the experimental measurements of Koedam and Kruithof [5]. This agreement between measurements and calculations can be partially justified by the fact that the previous distribution is the formal solution of the diffusion equation without sources terms. In this work we would like to examine the validity of 
this assumption, thus the following radial normalized distributions have been tested :

$$
\begin{array}{ll}
P_{1}(r)=1-\frac{r}{R} & P_{2}(r)=1-\left(\frac{r}{R}\right)^{2} \\
P_{4}(r)=1-\left(\frac{r}{R}\right)^{4} & P_{6}(r)=1-\left(\frac{r}{R}\right)^{6}
\end{array}
$$

Our results have shown that the use od $P_{2}$ instead of the Bessel shape leads to a good agreement with the experiment. However, the use of $P_{1}, P_{4}$ and $P_{6}$ shapes was averred in conflict with the experimental data. Furthermore, it is possible to simplify our calculations by using only averaged values along the discharge radius. Using a well known radial profile (i.e. Bessel distribution) it was quite easy to obtain these averaged quantities by analytical integration over the discharge tube cross section.

The previous assumptions lead to a set of coupled ordinary differential equations described below :

- Hg excited state continuity equations :

$$
\frac{\partial n_{\mathrm{r}}}{\partial t}=n_{\mathrm{e}}\left[\sum_{\forall \neq\left\{\mathrm{Hg}^{+}\right\}} n_{y} Z_{y}-n_{\imath} \sum_{\imath \neq 1} Z_{v v}\right]+\mathcal{A}_{\lambda}+\mathfrak{K}_{\mathrm{r}}
$$

where, $x \in\{\mathrm{m}, \mathrm{r}, \mathrm{s}, \mathrm{p}, \mathrm{u}\}, y \in\left\{\mathrm{g}, \mathrm{m}, \mathrm{r}, \mathrm{s}, \mathrm{p}, \mathrm{u}, \mathrm{Hg}^{+}\right\}$and $n_{\mathrm{e}}$ is the electron density.

The operator $\mathcal{A}_{\mathrm{x}}$ takes into account the contribution of the radiative transitions incoming $(+\operatorname{sign})$ and outgoing $(-\operatorname{sign})$ to/from the level $x$. Consulting figure 1 the following values are found for this operator:

\begin{tabular}{|cccccc|}
\hline$x$ & $\mathrm{~m}$ & $\mathrm{r}$ & $\mathrm{s}$ & $\mathrm{p}$ & $\mathrm{u}$ \\
\hline $\mathcal{A}_{x}$ & $\frac{n_{\mathrm{u}}}{\tau_{\mathrm{um}}}$ & $\frac{n_{\mathrm{u}}}{\tau_{\mathrm{ur}}}-\frac{n_{\mathrm{r}}}{\tau_{\mathrm{rg}}}$ & $\frac{n_{\mathrm{u}}}{\tau_{\mathrm{us}}}$ & $-\frac{n_{\mathrm{p}}}{\tau_{\mathrm{pg}}}-\sum_{y=\mathrm{m}, \mathrm{r}, \mathrm{s}} \frac{n_{\mathrm{u}}}{\tau_{\mathrm{uy}}}$ \\
\hline
\end{tabular}

where $\tau_{x y}$ is the effective radiative life-time of the transition $x \rightarrow y$, taking into account the radiation trapping in the plasma.

The operator $\mathcal{H}_{x}$ accounts for inelastic collisions between $\mathrm{Hg}$ atoms. This operator has nonzero values only in the case of $x=\mathrm{s}$ then $\mathcal{H}_{\mathrm{s}}=-n_{\mathrm{s}}^{2} Z_{\mathrm{sHg}}^{\mathrm{s}}$ where $Z_{\mathrm{sHg}}^{\mathrm{s}}$ corresponds to the collision rate of the reaction (A), previously cited in this paper.

- Ar excited state continuity equation :

$$
\frac{\partial n_{x}}{\partial t}=n_{\mathrm{e}}\left[n_{y} Z_{y x}-n_{x}\left(Z_{x y}+Z_{x \mathrm{Ar}^{+}}\right)\right]-\frac{n_{x}}{\tau_{x v}}
$$

where $x=\operatorname{Ar}^{*}$ and $y=\operatorname{Ar}$.

- Ion continuity equations and local plasma neutrality :

$$
\begin{gathered}
\frac{\partial n_{\mathrm{Hg}^{+}}}{\partial t}=D_{\mathrm{a}}^{\mathrm{Hg}^{+}} \nabla^{2} n_{\mathrm{Hg}^{+}}+n_{\mathrm{e}} \sum_{x} n_{x} Z_{x \mathrm{Hg}^{+}}+n_{\mathrm{s}}^{2} Z_{\mathrm{sHg}^{+}}^{\mathrm{s}} \\
\frac{\partial n_{\mathrm{Ar}^{+}}}{\partial t}=D_{\mathrm{a}}^{\mathrm{Ar}^{+}} \nabla^{2} n_{\mathrm{Ar}^{+}}+n_{\mathrm{e}} n_{y} Z_{\mathrm{y} \mathrm{Ar}^{+}} \\
n_{\mathrm{e}}=n_{\mathrm{Hg}^{+}}+n_{\mathrm{Ar}^{+}}
\end{gathered}
$$

where $x \in\{\mathrm{g}, \mathrm{m}, \mathrm{r}, \mathrm{s}, \mathrm{p}, \mathrm{u}\}, y=\mathrm{Ar} *$ and $D_{\mathrm{a}}^{\mathrm{Ar}^{+}}, D_{\mathrm{a}}^{\mathrm{Hg}^{+}}$are the ion diffusion coefficients. 
- Electronic energy conservation equation :

$$
\frac{\partial U_{\mathrm{e}}}{\partial t}=n_{\mathrm{e}}\left[-\kappa \nu_{\text {coll }}\left(U_{\mathrm{e}}-U_{\mathrm{g}}\right)+q \mu_{\mathrm{e}} E^{2}+\sum_{y \neq x} n_{x} Z_{x y} \Delta U_{x y}\right]
$$

$x \in\left\{\mathrm{g}, \mathrm{m}, \mathrm{r}, \mathrm{s}, \mathrm{p}, \mathrm{u}, \mathrm{Ar}, \mathrm{Ar}^{*}\right\}$ and $y \in\left\{\mathrm{g}, \mathrm{m}, \mathrm{r}, \mathrm{s}, \mathrm{p}, \mathrm{u}, \mathrm{Hg}^{+}, \mathrm{Ar}, \mathrm{Ar}^{*}, \mathrm{Ar}^{+}\right\} . U_{\mathrm{e}}$ is the average electron energy, $U_{\mathrm{g}}$ is the average thermal energy of the neutral atoms and $\Delta U_{x y}=U_{y}-U_{x}$ is the energy gap between the levels $x$ and $y$. If the transition leads to the ionization $\left(y \in\left\{\mathrm{Hg}^{+}, \mathrm{Ar}^{+}\right\}\right)$then wall losses should be considered by means of the relation : $\Delta U_{x y}=\Delta U_{x y}-5 U_{\mathrm{e}} / 2$. Other symbols in the above equation are : $\nu_{\text {coll }}$ the electron-argon elastic collision frequency, $\mu_{\mathrm{e}}$ the electron mobility and $\kappa$ the fractional energy loss per electron per elastic collision. The term corresponding to $\mathrm{Hg}$ associative ionization (reaction $\mathrm{A}$ ), proportional to $n_{\mathrm{s}}^{2}$, is negligible because of the very small rate coefficient corresponding to this reaction (several orders of magnitude less than electron-atom collision rates).

- Ohm's law :

$$
I=2 \pi q E \int_{0}^{R} n_{\mathrm{e}} \mu_{\mathrm{e}} r \mathrm{~d} r
$$

where $E$ is the axial electric field strength and $q$ the elementary electron charge (absolute value).

In these equations, $Z_{x y}$ are the corresponding rate coefficients calculated from the relevant $Q_{x y}$ inelastic cross sections. In the case of excitation from the $\mathrm{Hg}\left(6^{1} \mathrm{~S}_{0}\right)$ state we used the Rockwood's data [6]. Excitation cross sections for the excited $\mathrm{Hg}$ levels are given by Winkler [14] and cross sections for the ionization from these levels are obtained from Vriens's [7] semi-empirical formula. Data concerning argon, have been given by Zissis [3]. All reactions rate for the 2 nd kind collisions are determined by using the Klein-Rosseland's relation based on the micro-reversibility principle. The effective radiative life-times, $\tau_{x y}$, and data concerning the two resonance mercury lines $(254 \mathrm{~nm}$ and $185 \mathrm{~nm})$ used in this study are given by Holstein's [8] theory, Bernat's calculations [9] and Van de Weijer's experimental values [10].

The knowledge of the Electron Energy Distribution Function is of prime importance. All transport coefficients depend on the one hand, on it and the transition rates are very sensitive to the EEDF choice on the other hand. For example, the relative difference between transition rates calculated by using different EEDF approximations can reach $90 \%$ in the case $7^{z^{-}} \rightarrow 6^{1} S_{0}$ transition [17]. In the present work the Maxwell-Boltzmann classical EEDF has been mainly used, this assumption simplifying considerably the calculations. However, in the case of steady-state calculations we have examined the influence of a non-Maxwellian EEDF shape. For this attempt, the Lagushenko and Maya's [4] semi-empirical analytical solution of the Boltzmann equation has been implemented as EEDF. The results obtained using this function are clearly more realistic but the computer time, in the case of ac operating mode, becomes prohibitive (several hours of calculations on a HP-9000/330 computer).

\section{Results.}

STEADY STATE OPERATING MODE $(\partial / \partial t=0)$. - Firstly, we compare our results with experimental data in a dc discharge in order to validate our model. Indeed, such studies are more common. We use experimental data given by Damelincourt [2] and Koedam [5] for the $\mathrm{Hg}\left(6^{3} \mathrm{P}_{0}, 1,2\right)$ densities. These measurements have been re-iterated recently by Bernat [9]. 
Our main intention during this phase is to evaluate the validity of certain assumptions systematically adopted by several authors.

Our initial results were not in good agreement with those given by experiments. This could be explained by the fact that in a first approach we neglected $\mathrm{Hg}$ associative ionization as well as Ar excitation and ionization. Furthermore, we considered the imprisonment lifetime gave by Holstein's theory. As we have shown in the previous part of this paper the radial distribution of the charged species could severely influence the emitted power of the $\mathrm{Hg}-254 \mathrm{~nm}$ if $T_{\mathrm{cs}}$ was greater than $40{ }^{\circ} \mathrm{C}$. In view of the overall of the results, we suppose a Bessel shape radial distribution for the charged and excited species and a better agreement is obtained. Furthermore, the comparison of our results for the $\mathrm{Hg}$ resonance radiation output in the case of a typical low pressure discharge with those given by more complete radial calculations carried out by Zissis [16] leads to a satisfactory agreement.

As illustrated in figure 3 , we demonstrate the importance of $\mathrm{Hg}$ associative ionization on the $\mathrm{Hg}\left(6^{3} \mathrm{P}_{0,1,2}\right)$ populations at higher cold spot temperature range. Moreover, the validity of Holstein's theory influences the middle temperature range. Besides, argon excitation and ionization weight is not negligible mainly in the low cold spot temperatures.

As far as the influence of the escape factor of the mercury resonance lines are concerned, we tested initially values deduced from Holstein's theory [8], and then those of Bernat [9] and Weijer [10] for the $\mathrm{Hg}-254 \mathrm{~nm}$ and $185 \mathrm{~nm}$ lines respectively. The second set of values, $30 \%$ or $40 \%$ lower than Holstein's is in better agreement with the values given by experiments. Figure 3 shows that this choice leads to a sensitive decrease of the density populations.

A very common hypothesis is to negglect the inelastic scattering between excited $\mathrm{Hg}$ atoms. In fact, for $\mathrm{Hg}$ partial pressures less than $10 \mathrm{~m}$ Torr, the atomic ratio $\left[\mathrm{Hg}^{*}\right] /[\mathrm{Hg}]$ remains less than $1 \%$, thus the probability of $\mathrm{Hg}^{*} / \mathrm{Hg}^{*}$ inelastic scattering seems to be negligible. However, it is quite hazardous to neglect these collisions when $\mathrm{Hg}$ partial pressure becomes important. As shown, the reaction (A) leads to a decrease of the $\mathrm{Hg}\left(6^{3} \mathrm{P}_{2}\right)$ density. However, we do not dispose precise information on the nature of these interactions and the published cross section data are very uncertain. These cross section values find in the literature vary between $1 \AA^{2}$ and $100 \AA^{2}$. As shown in figure 3, a good agreement between calculation and experimental data is obtained when this cross section approaches $50 \AA^{2}$.

For lower values of $T_{\mathrm{cs}}$, when mercury partial pressure becomes very low (some $100 \mu$ Torrs), without including Ar excitation and ionization in our model, significant deviations between calculation and experimental data have been observed. By including these phenomena and choosing a suitable Ar resonance radiation trapping factor our results, as shown in figure 3, fit well the excited states population. Furthermore, as has been shown elsewhere [18] the influence of the Ar is of a prime importance not only for population calculations but also for the determination of the axial electric field strength and the electron temperature in the discharge plasma.

As shown in figures 4 and 5 the agreement between our calculations and experimental data for the $\mathrm{Hg}-254 \mathrm{~nm}$ resonance line emission intensity and the axial excited state densities of the $\mathrm{Hg}\left(6^{3} \mathrm{P}_{0,1,2}\right)$ excited levels as function of the cold spot temperature is quite satisfactory. The discrepancies observed in the other author's results [13-15] can be explained by the fact that they do not account for $\mathrm{Hg}$ associative ionization, and besides, most of them use the Holstein's theory for the determination of the escape factors. Moreover, they do not consider argon as an active species.

The above considerations validate our model in steady-state operating mode. The better agreement has been obtained by using the following combination of parameters :

- Escape factors :

$$
\tau_{\mathrm{rg}}(\mathrm{Hg}-254 \mathrm{~nm}): \text { as given by Bernat's calculations [9] }
$$



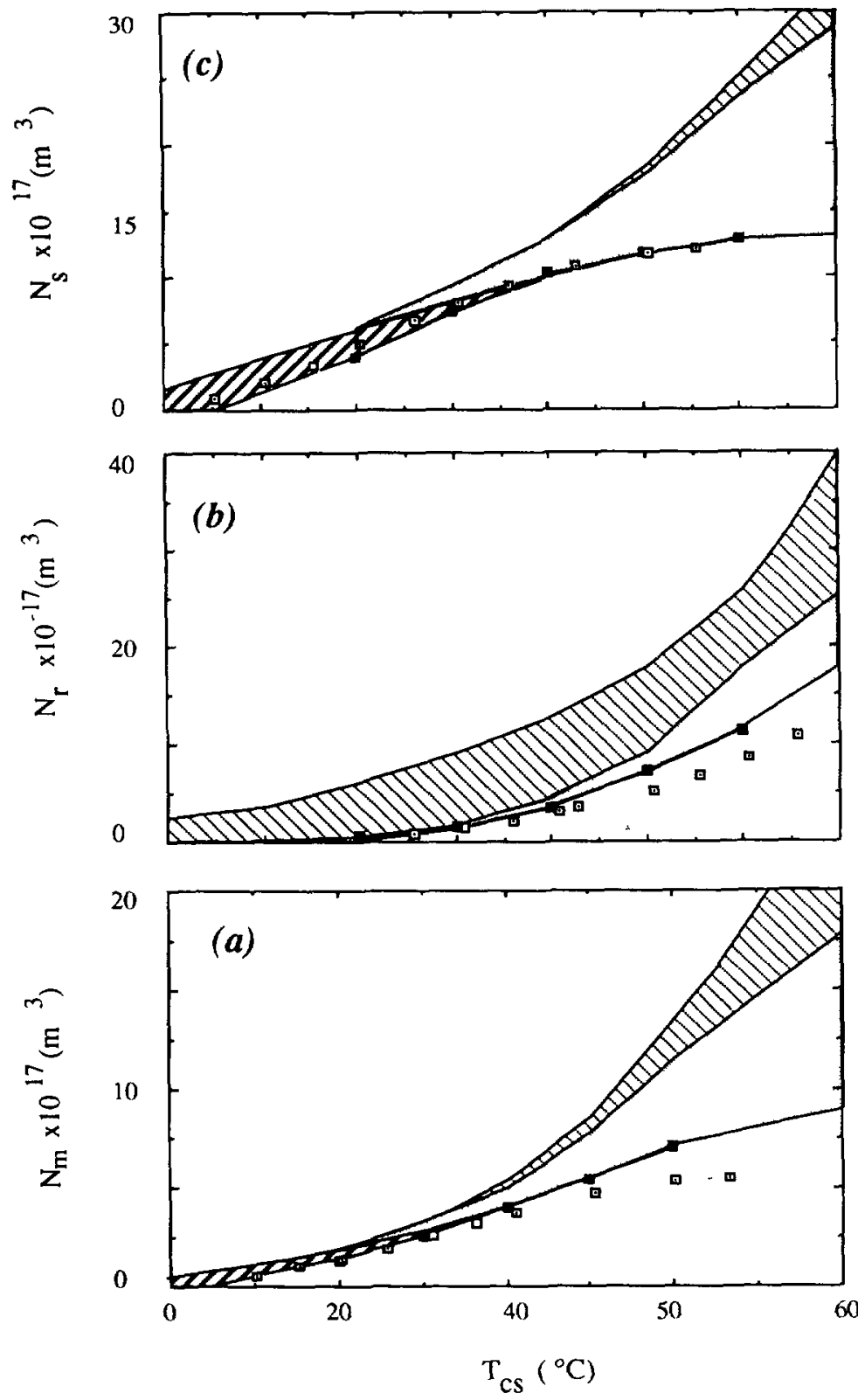

Fig. 3. - Influence of different physical processes on the mercury excited state populations (curve a : $\mathrm{Hg}-6^{3} \mathrm{P}_{0} ; \mathrm{b}: \mathrm{Hg}-6^{3} \mathrm{P}_{1} ; \mathrm{c}: \mathrm{Hg}-6^{3} \mathrm{P}_{2}$ ) as function of the cold spot tube temperature. Shadowed areas correspond to the influence domain of the following processes: $\mathrm{Hg}$ associative ionization (reaction a) with cross section $\sigma$ varying from 0 to $50 \AA^{2} ; \mathbb{Z}$ Ar excitation and ionization with excited state effective life time, $\tau_{\mathrm{Ar}}$ varying from 0 (excitation and ionization excluded) to 5 times the experimental value given by [11]; $\mathrm{Hg}$ resonance radiation escape factors, $\tau_{\mathrm{rg}}$ and $\tau_{\mathrm{pg}}$ varying from the Holstein's theory to experimental values given by [9] and [10] respectively, - - our final calculations with $\sigma=50 \AA^{2}, \tau_{\mathrm{rg}}$ from [9], $\tau_{\mathrm{pg}}$ from [10], $\tau_{\mathrm{Ar}}$ from [11], including Ar excitation and ionization. Experimental data $(\square)$ are from reference [2]. 


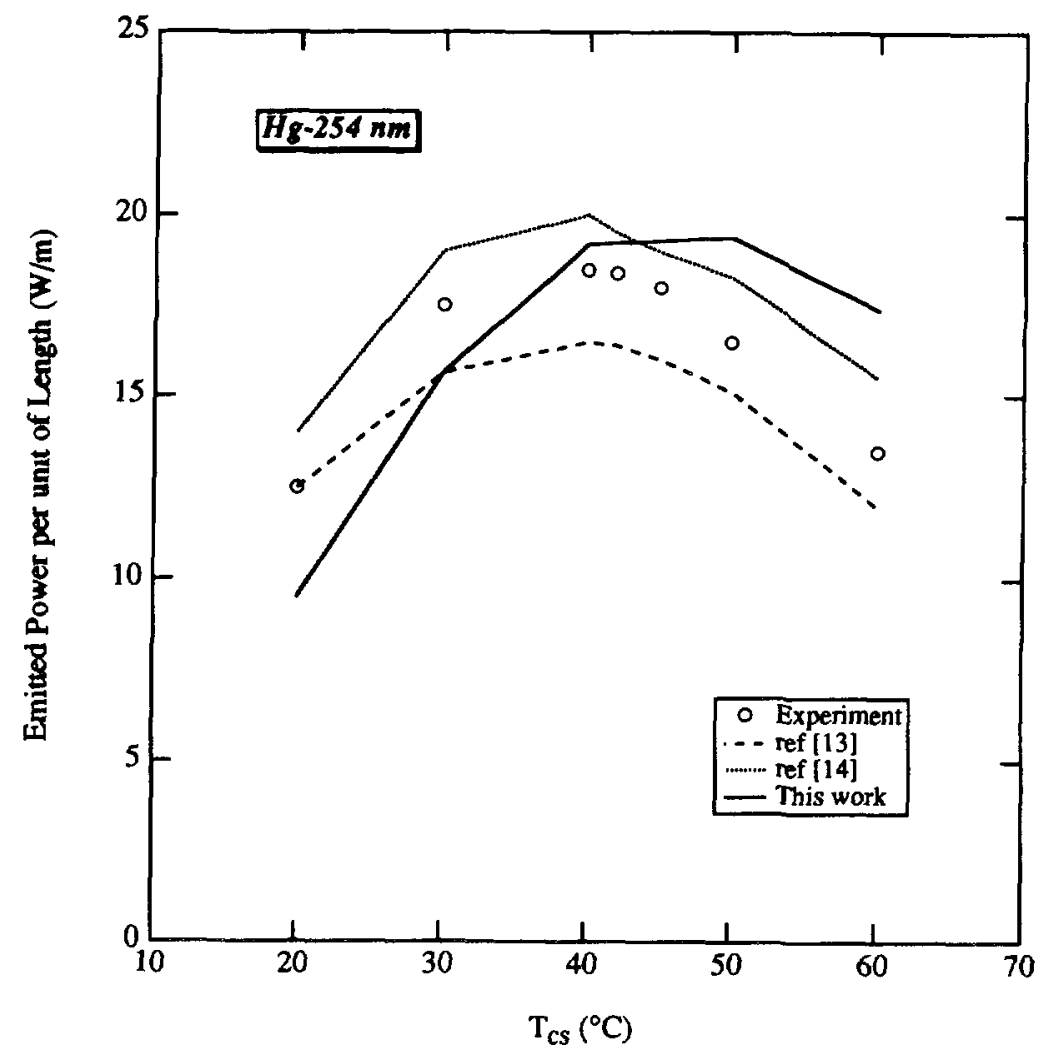

Fig. 4. - Comparison between several calculated values (lines) of the emitted power at $254 \mathrm{~nm}(\mathrm{Hg}$ resonance line) per unit of positive column length and the experimental data (symbols). Experimental data are from reference [2].
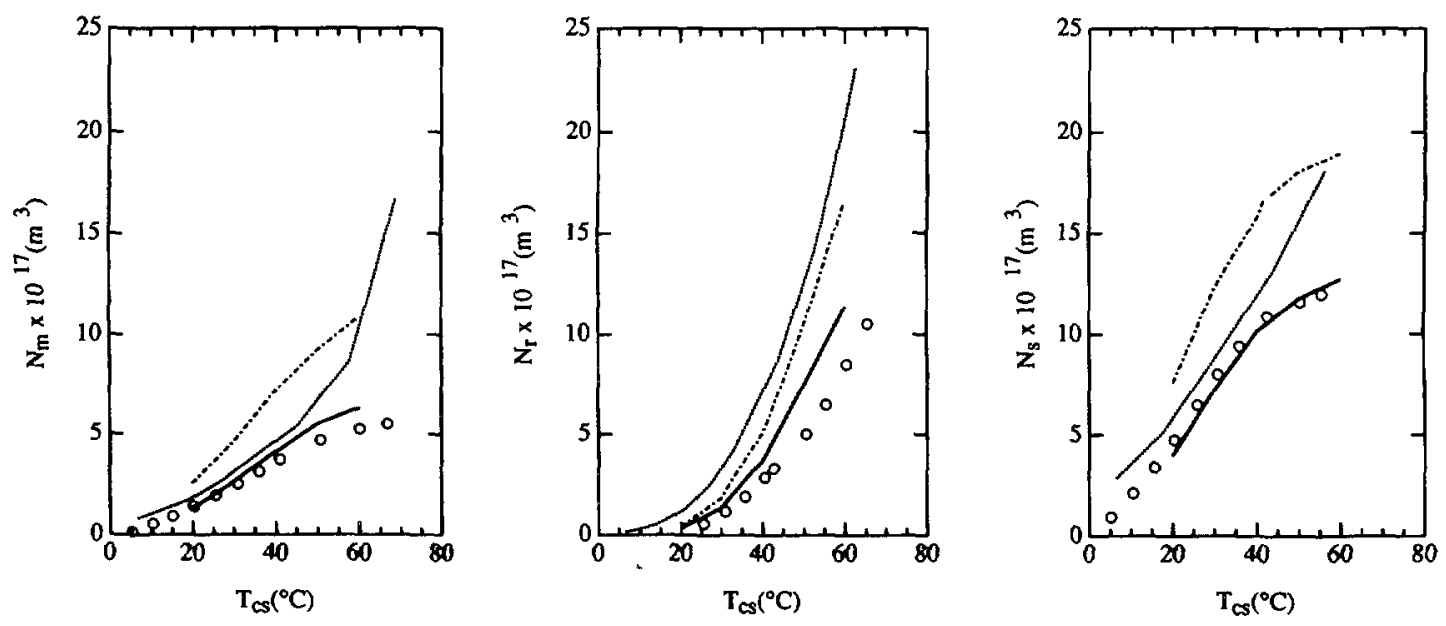

Fig. 5. - Comparison between several calculated values of excited-state densities of the $\mathrm{Hg}\left(6^{3} \mathrm{P}_{0,1,2}\right)$ atomic levels and experimental data as a function of the cold spot temperature. ( 0 ) experimental data [5] ; calculations : $(----)$ : reference $[14] ;(-.-)$ : reference $[15] ;(-)$ : this work. 
$\tau_{\mathrm{pg}}(\mathrm{Hg}-185 \mathrm{~nm}):$ as given by Weijer [10]

$\tau_{\mathrm{Ar}}(\mathrm{Ar}-105 \mathrm{~nm}):$ as given by data in reference [11].

- $\mathrm{Hg}$ associative ionization cross section (reaction $\mathrm{A}$ ) :

$$
\sigma=50 \AA^{2}
$$

SINUSOIDAL OPERATING MODE $(\partial / \partial t \neq 0)$. - Experimental works carried out by Damelincourt [2] and Bernat [9] demonstrate the great difficulties to interpret excited state populations leading from measurements of the $\mathrm{Hg}-254 \mathrm{~nm}$ absorption in the discharge plasma. When the frequency is about $0.1 \mathrm{kHz}$ and lower, the wave form of the current is not a true sine curve (Fig. 6). For the above reasons we use the experimental data of the relative flux of the $\mathrm{Hg}$ $254 \mathrm{~nm}$ resonance line in order to validate our calculations. These measurements are direct and do not need any interpretation calculations. The good agreement between data and calculations shown in figure 6. However, there is a problem at low frequency. At the very beginning of the half period of the current, the experimental values present an important increase followed by a sensible decrease.

For the other quantities such as densities, electric field, electron temperature, our calculations do not reproduce experimental results quantitatively. However, they fit the experimental data qualitatively well. Figure 7 gives the calculation results and the corresponding experimental values. These quantitative discrepancies could be explained as following.

The rapid increase of $n_{x}, E$ and $T_{\mathrm{e}}$ observed at the beginning of the half-period due to the fact that there is an «extinction » of the discharge corresponding to a very low electron density. The field strength $E$ must increase in order to maintain the current in the discharge. This " heats" the electrons and $T_{\mathrm{e}}$ increases as well. At the beginning of the half period, there are not enough excited species and electrons. Only inelastic collisions between electrons and $\mathrm{Hg}$ ground state atoms take place and they populate directly the higher excited levels. While $n_{\mathrm{e}}$ remains low, $\mathrm{Hg}\left(6^{3} \mathrm{P}_{0}\right)$ and $\mathrm{Hg}\left(6^{3} \mathrm{P}_{2}\right)$ cannot depopulate because they can only decay by 2nd kind collisions with electrons. In opposition, because of its rapid decay by radiative transitions, the population of the $\mathrm{Hg}\left(6^{3} \mathrm{P}_{1}\right)$ resonant level does not increase significantly at the beginning of the half period.

As shown in figures 8 and 9 , representing the time-average values of the electric field and the electron density population over a period, there is a good agreement between calculated values and experimental data.

\section{Conclusion.}

A first study in the frame of the steady state, allows us to evaluate the "weight " of certain physical phenomena in this type of modelling. We assess how much it is of prime importance to consider $\mathrm{Hg}-\mathrm{Hg}$ inelastic scattering as well as the excitation and ionization of argon in our discharge plasma. The first point, often discarded by many authors, has quite an appreciable influence on the different discharge parameters. Besides, the determination of the escape factors for the $\mathrm{Hg}-254 \mathrm{~nm}$ and $185 \mathrm{~nm}$ resonance lines are not at least inconsequential.

In the case of sinusoidal excitation, we deal with a more complete study about frequency dependence of excited state and ion densities, electronic temperature and axial electric field. We use the experimental data of the relative flux of the $\mathrm{Hg}-254 \mathrm{~nm}$ resonance line in order to validate our calculations. A good agreement between experimental data and calculations is found. 

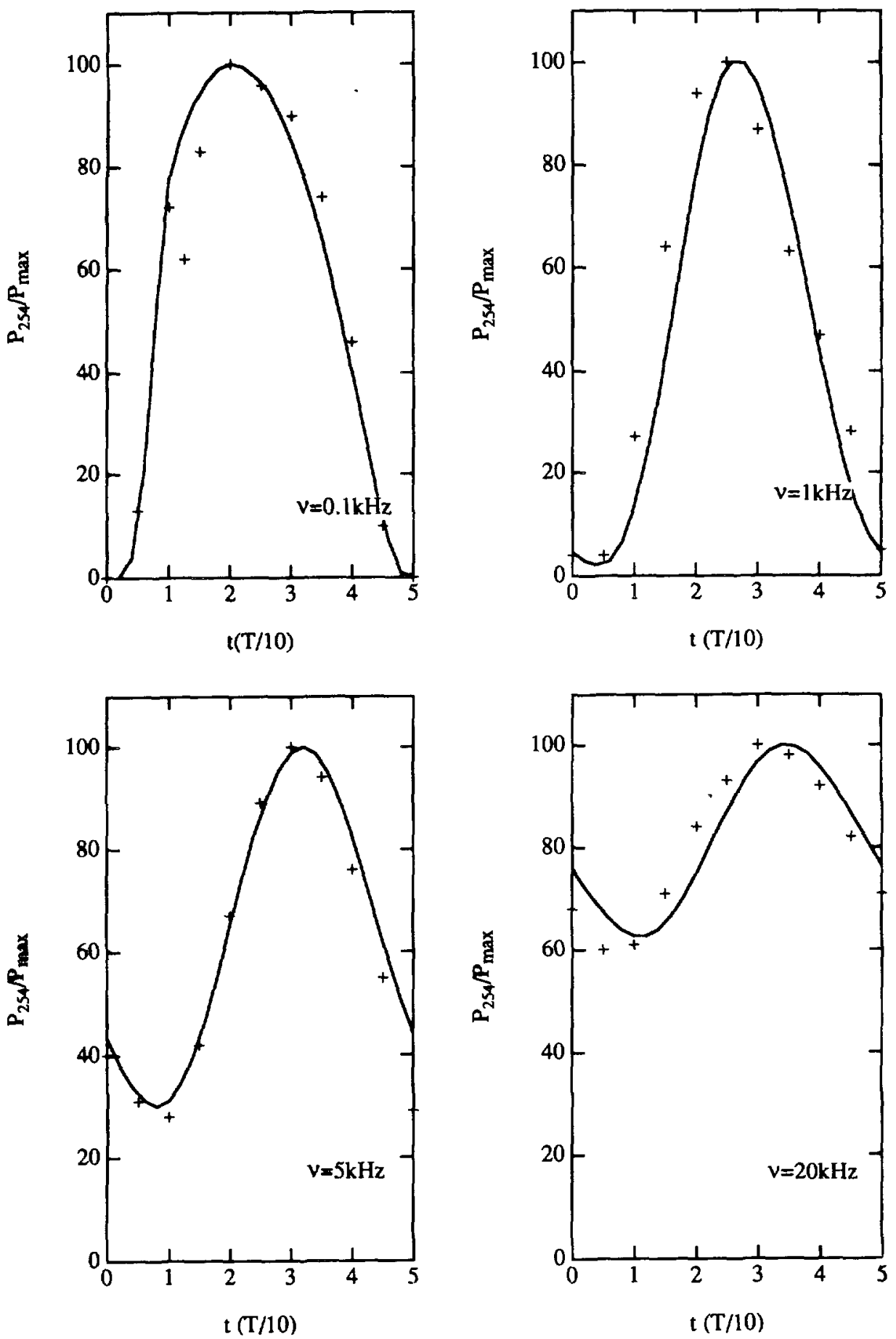

Fig. 6. - Comparison between calculated values of the relative emitted power at $254 \mathrm{~nm}$ and the experimental data over one half-period of the electric current, for different frequencies. This work: $(-)$; experimental data : $(+)[2]$. 


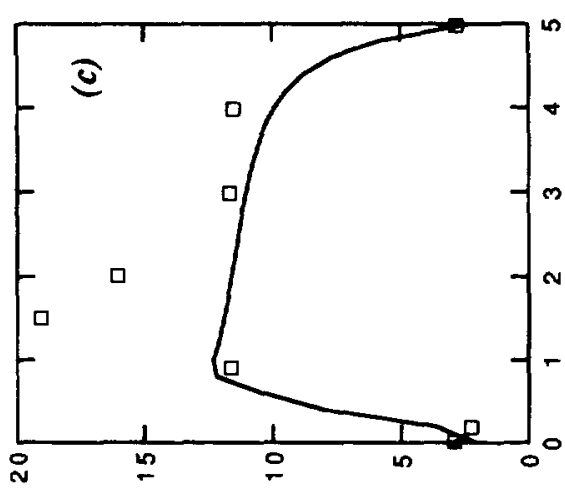

(X) ${ }_{\varepsilon} 01 x^{\circ} \mathrm{J}$

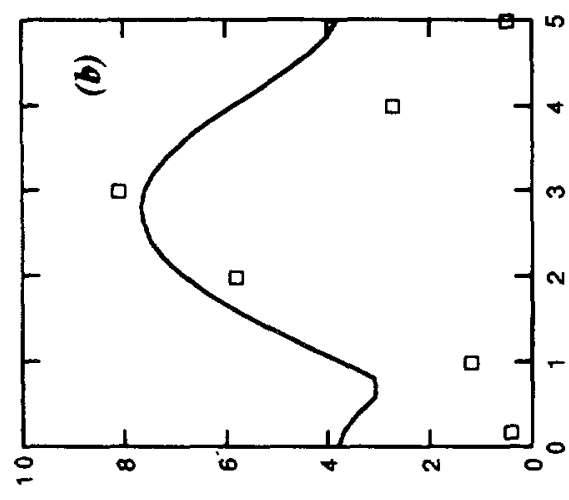

$\left(_{\varepsilon^{-}} \cdot \mathrm{ll}\right)_{41} .01 \mathrm{X}^{2} \mathrm{~N}$

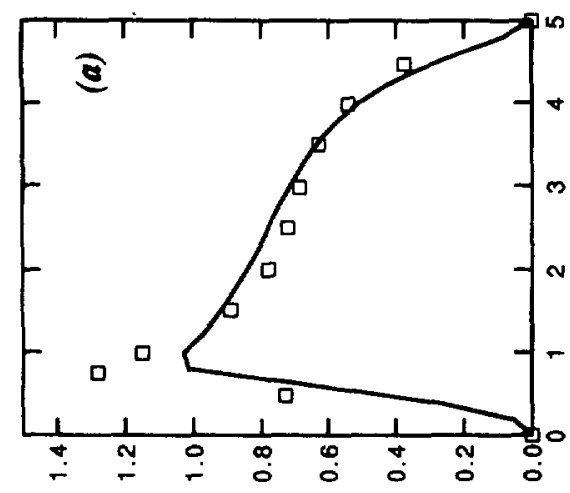

(แั/ก) $\exists$

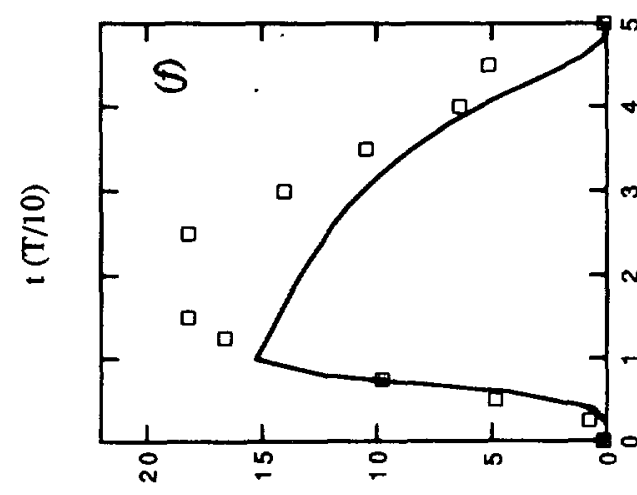

$\left(\varepsilon^{-} \cdot w\right)_{L L}-0 I x^{8} \mathrm{~N}$
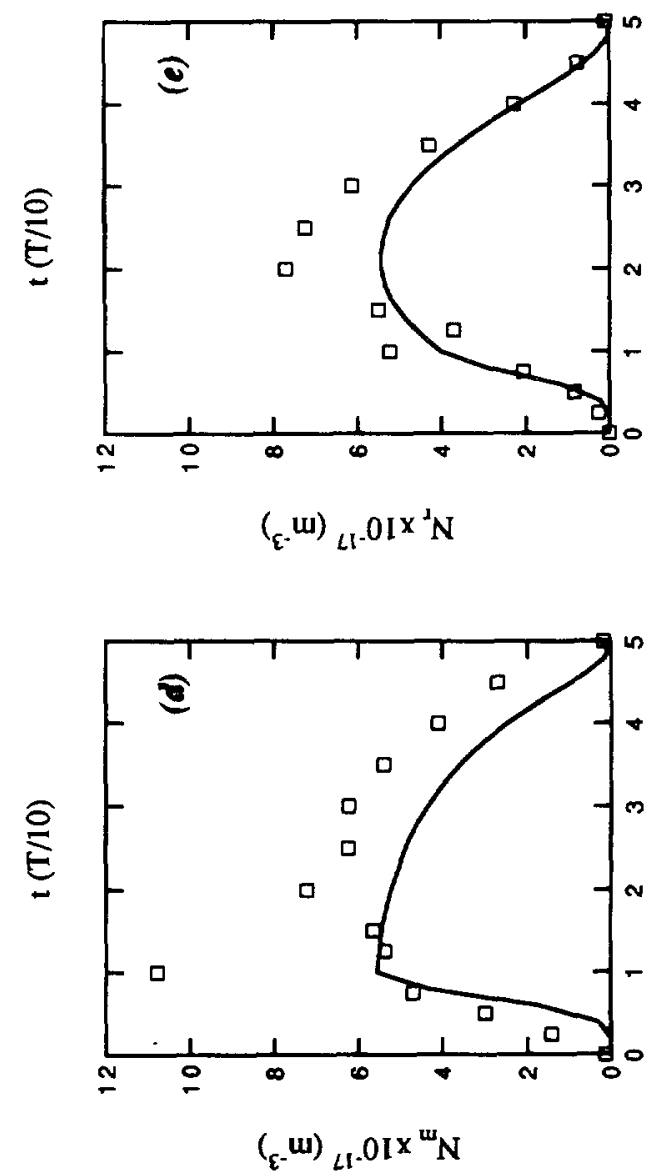

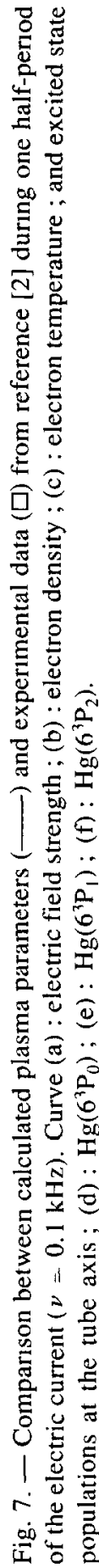



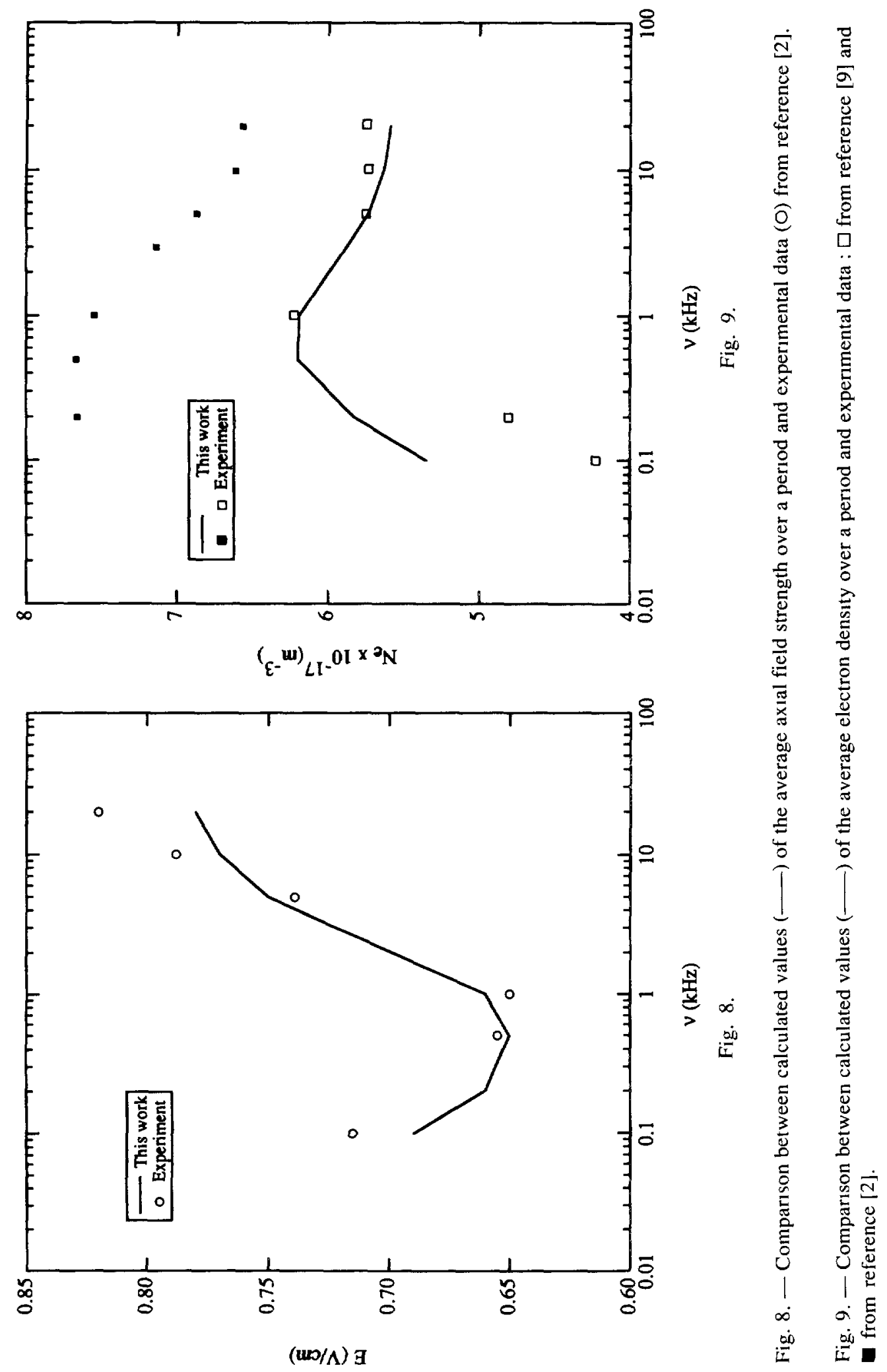


\section{Acknowledgments.}

The authors are greatly indebted to Professor J. J. Damelincourt for his extremely useful remarks and the unflagging supervision of this work. We would also like to thank $\mathrm{K}$. Charrada and L. Sévier for their contribution through many discussions and N. Sewraj for his assistance in the realisation of this paper.

\section{References}

[1] Campbell J. H., Ill. Eng. (1960) 247.

[2] Damelincourt J. J., Thèse d'état, Université Paul Sabatier (Toulouse, 1973).

[3] Zissis G., Thèse, Université Paul Sabatier (Toulouse, 1990).

[4] Lagushenko R., MaYa J., J. I.E.S. 14 (1986) 306.

[5] Koedam M., Kruithof A. A., Physica 28 (1962) 80.

[6] Rockwood S. D., Phys. Rev. A 8 (1973) 2348.

[7] Vriens L., Keuser R. A. J., Ligthart F. A. S., Phys. Rev. 22 (1980) 940.

[8] Holstein T., Phys. Rev. 72 (1947) 1212.

[9] Bernat I., Thèse, Université Paul Sabatier (Toulouse, 1991).

[10] Van de Weijer P., Cremers R. M. M., J. Appl. Phys. 53 (1982) 1401.

[11] Payne M. G., Talmage J. E., Hurst G. S., Wagner E. B., Phys. Rev. 9 (1970) 1050.

[12] VerweIJ W., Philips Res. Suppl. (1961) 2.

[13] Waymouth J. F., Bitter F., J. Appl. Phys. 27 (1956) 122.

[14] Winkler R. B., Wilhelm J., Winkler R., Ann. Phys 7 (1983) 90.

[15] Dakin J. T., J. Appl. Phys. 20 (1986) 563.

[16] Zissis G., Bénétruy P., Bernat I., Phys. Rev. A 45 (1992) 1135.

[17] Yousfi M., Zissis G., AlkaA A., Damelincourt J. J., Phys. Rev. A 42 (1990) 978.

[18] Plagnol V., Zissis G., Ziane M., 1991, in proc. XXth Int. Conf. Phen. Ion. Gas, M. Palleschi and L. Vaselli Eds., Pisa 6, p. 1283. 
Articles Réguliers 